\title{
CARE: Context-awareness for elderly care
}

\author{
Simon Klakegg ${ }^{1}$ (Dennedy Opoku Asare ${ }^{2} \cdot$ Niels van Berkel $^{3} \cdot$ Aku Visuri $^{2} \cdot$ Eija Ferreira $^{2} \cdot$ Simo Hosio $^{2}$. \\ Jorge Goncalves ${ }^{4} \cdot$ Hanna-Leena Huttunen ${ }^{2} \cdot$ Denzil Ferreira $^{2}$
}

Received: 27 May 2020 / Accepted: 17 November 2020

(c) The Author(s) 2021

\begin{abstract}
We present CARE, a context-aware tool for nurses in nursing homes. The system utilises a sensors infrastructure to quantify the behaviour and wellbeing (e.g., activity, mood, social and nurse interactions) of elderly residents. The sensor data is offloaded, processed and analysed in the cloud, to generate daily and long-term summaries of residents' health. These insights are then presented to nurses via an Android tablet application. We aim to create a tool that can assist nurses and increase their awareness to residents' needs. We deployed CARE in a local nursing home for two months and evaluated the system through a post-hoc exploratory analysis and interviews with the nurses. The results indicate that CARE can reveal essential insights on the wellbeing of elderly residents and improve the care service. In the discussion, we reflect on our understanding and potential impact of future integrated technology in elderly care environments.
\end{abstract}

Keywords Sensors $\cdot$ Data analysis $\cdot$ Elderly care $\cdot$ System design

\section{Introduction}

With a significant increase in the elderly population, nurses are experiencing growing workloads and stress levels. The profession is suffering from large turnover rates, leading to an influx of new personnel [1]. This is problematic as the newcomers often lack relevant patient information to ensure satisfying continuity of care [2]. Furthermore, nurses have a very mobile occupation; more than $50 \%$ of their time is spent on the move [3]. In such a hectic environment, it is challenging for nurses to remember all the details of their patients [3]. Detecting small health or behavioural changes among patients, potentially accumulating over the long term into more serious conditions, can be difficult without the appropriate tools [4].

A critical component of the nurses' workday is a daily meeting called 'the handover' [5]. In this meeting, essential information of the day-to-day health of patients is transferred between staff members. However, this handover is often done informally and inconsistently without a systematic approach [6].
Simon Klakegg
simon.klakegg@live.no
Kennedy Opoku Asare
kennedy.opokuasare@oulu.fi
Niels van Berkel
nielsvanberkel@cs.aau.dk
Aku Visuri
akuvisur@oulu.fi
Eija Ferreira
eija.ferreira@oulu.fi
Simo Hosio
simo.hosio@oulu.fi
Jorge Goncalves
jorge.goncalves@unimelb.edu.au
Hanna-Leena Huttunen

hl.huttunen@oulu.fi

Denzil Ferreira

zil.Ferreira@oulu.fi

1 Tellu IoT, Asker, Norway

2 University of Oulu, Pentti Kaiteran katu 1, 90570 Oulu, Finland

3 Aalborg University, Fredrik Bajers Vej 7K, 9220 Aalborg, Denmark

4 The University of Melbourne, Parkville VIC 3010, Melbourne, Australia 
Eldery care providers are now adapting the use of technology to alleviate some of the aforementioned challenges. For instance, systems which notify nurses on patient events [7] can increase efficiency and better utilisation of resources. Logging vital signs and automatically sharing the data with various health instances can be simplified with wireless sensor networks [8]. Also, location-aware fall detection [9] can increase resident safety. Given the rapid innovation of sensor hardware, network infrastructure and smart devices over the past few years, ambient assisted living systems can now prolong the time elderly live independently at home [10]. However, there is still a scarcity of $i n-s i t u$ tools that can support nurses' daily tasks in a residential care environment $[11,12]$. The majority of previous work has focused on supporting independent living. In this paper, we explore how we can integrate a context-aware sensor system (CARE) in a nursing home and what the benefits are. The contribution of our work is three-fold:

- We integrate all components of CARE in a collaborating nursing home and demonstrate how to enable pervasive sensing without interfering residents or nurses.

- We conduct a 2-month user study to evaluate the benefits of CARE and improve our understanding of the sociocultural context in the nursing home.

- We discuss how technology can empower nurses and improve the care service.

\section{Related work}

\subsection{Elderly care}

Global life expectancy has risen in the past decades, resulting in a worldwide growing demand on countries' healthcare systems [13]. One in five Americans is calculated to be 65 years or older by 2030 . The old-age dependency ratio is projected to steadily increase in the coming years [14]. Public long-term care expenditures for Organisation for European Economic Co-operation countries averaged $1.4 \%$ of gross domestic product in 2009 , a number expected to at least double by 2050 [15]. According to a report commissioned by the World Health Organization, there will be a lack of 12.9 million healthcare workers by 2035 and the current shortage is already mounting to 7.2 million [16]. The report also highlights innovative technology as a promising solution to these challenges. As the burden on staff in elderly care increases (e.g., higher workload, stress), work satisfaction declines which lead to occupational burnout [17]. This is a major cause for the high turnover rates in elderly care. Since the nursing homes experience a shortage of workers, there is an influx of new temporary staff (e.g., rotating nurses, students). These nurses usually lack important patient-specific information and experience, which lowers the quality of the healthcare service [18].

An estimated 20\% of old adults receive care at an institution, while $30 \%$ receives care at home in European countries $[19,20]$. The amount of consumers in both care contexts (i.e., home and institution) is expected to surge, and it is vital to developed solutions for each. Elderly care is especially demanding given the wide range of severe health issues that patients struggle with.

\subsection{Nurse workflow}

A considerable effort is made by healthcare providers to acquire new Information and Communication Technology (ICT) to assist their personnel. Yet, a majority of ICT implementations fail due to end-user resistance caused by unrealistic expectations and improper training [21]. To examine the workflow of nurses, their activity and location were recorded using direct observation in an extensive study [22]. Several of the daily tasks could be characterised as frequent and short. Furthermore, this type of work environment inhibited critical thinking and care planning. An investigation of workflows in social organisations (e.g., care centres, hospitals) indicate that they are nonlinear and this must be accounted for in the design of new technology [23].

In the dynamic medical environment healthcare personnel often utilise workarounds or shortcuts (e.g., patient A falls, so check-up on patient $B$ is postponed). There is a need for technological artefacts that can assist the user to plan the care in these situations [24]. A reoccurring practice in nursing homes is the handover, defined as: "Transfer of professional responsibility and accountability for some or all aspects of care for a patient, or groups of patients, to another person or professional group on a temporary or permanent basis" [25]. Handovers in elderly care have been described as high-speed, cryptic and challenging for new personnel [26]. They directly impact patients' safety as crucial information is discussed and shared during the meeting (e.g., a patient has not received medication). The handover is considered a priority by the world health organization and is included in the High 5s [27] - a project intending to reduce the magnitude of five key patient safety challenges worldwide.

\subsection{Elderly care technology}

The imminent burden of an aging population is driving researchers to focus on innovation and development of technology for elderly care. Consequently, a variety of valuable applications that can aid patients or nurses have started to emerge. For instance, sensors embedded in everyday objects can detect when old adults fall, which is a likely cause of death [28]. Hsu et al. [29] developed a clustering model for 
detecting abnormal behaviour (e.g., not eating, not using toilet, lack of movement) using data from Radio-Frequency Identification (RFID) tags. When outliers are identified, nurses or family members can be warned and asked to inspect the elderly. Quantifying nurses' activity and behaviour can help management understand their workflow and find areas of improvement such as inefficient task flow [30]. Data from smart devices can be analysed and used to empower a multitude of personal health applications or third-party monitoring services [31]. SensCare [32] is a system that measures the elderly users' activities (e.g., exercise, sleep, eating) using a smartphone and generate daily summaries. As old adults can have a limited ability to quantify their own life, this information can be used by nurses for accurate health interpretations. ICT in elderly care has the potential to reduce operational costs [33], for instance through telehealth which can lower hospital visits, care time and improve care quality for old adults living independently [34].

However, these types of system may not be suitable for nursing homes which always have nurses available around the clock. In this article, we mainly focus on assistive tools for nurses in nursing homes, which increases context-awareness using sensor data. The limited body of work which exists for this research scope includes systems for nurse notification [35], activity monitoring [30, 36], vital sign monitoring [8], fall detection [9] and wellbeing tracking [37]. Most of these systems focus on a specific use case (e.g., fall detection, measuring activity), with many conceptual features and evaluations are limited to controlled studies.

\section{Preliminary work}

The system we integrate into the nursing home and evaluate in this paper builds on previous work conducted in the CARE research project. In this section, we summarise three components that have previously been completed. These are relevant and will help to contextualise the work in this article.

1 Care metrics: To inform the nurses on patients' needs, we first had to establish what information was useful to the nurses. Through a 5-day long study in a nursing home, we established 18 care metrics which can be defined as: "a unit of information that may be measured and observed as part of the elderly care work practices" [38].

2 CARE app: We designed an Android app which could communicate the care metrics to nurses Fig. 1. The main view contains a list of all the patients with up to four care metrics that require attention (e.g., low activity, bad mood). A resident can be pressed to view more details on medical status (health record, allergies etc.), family members (name, call and text functionality), personality (likes, dislikes etc.) and health history (long term graph representations of care metrics). The app also has a handover mode which can be used during the daily meeting. It loops through each resident while displaying relevant data summaries and enables the nurses to
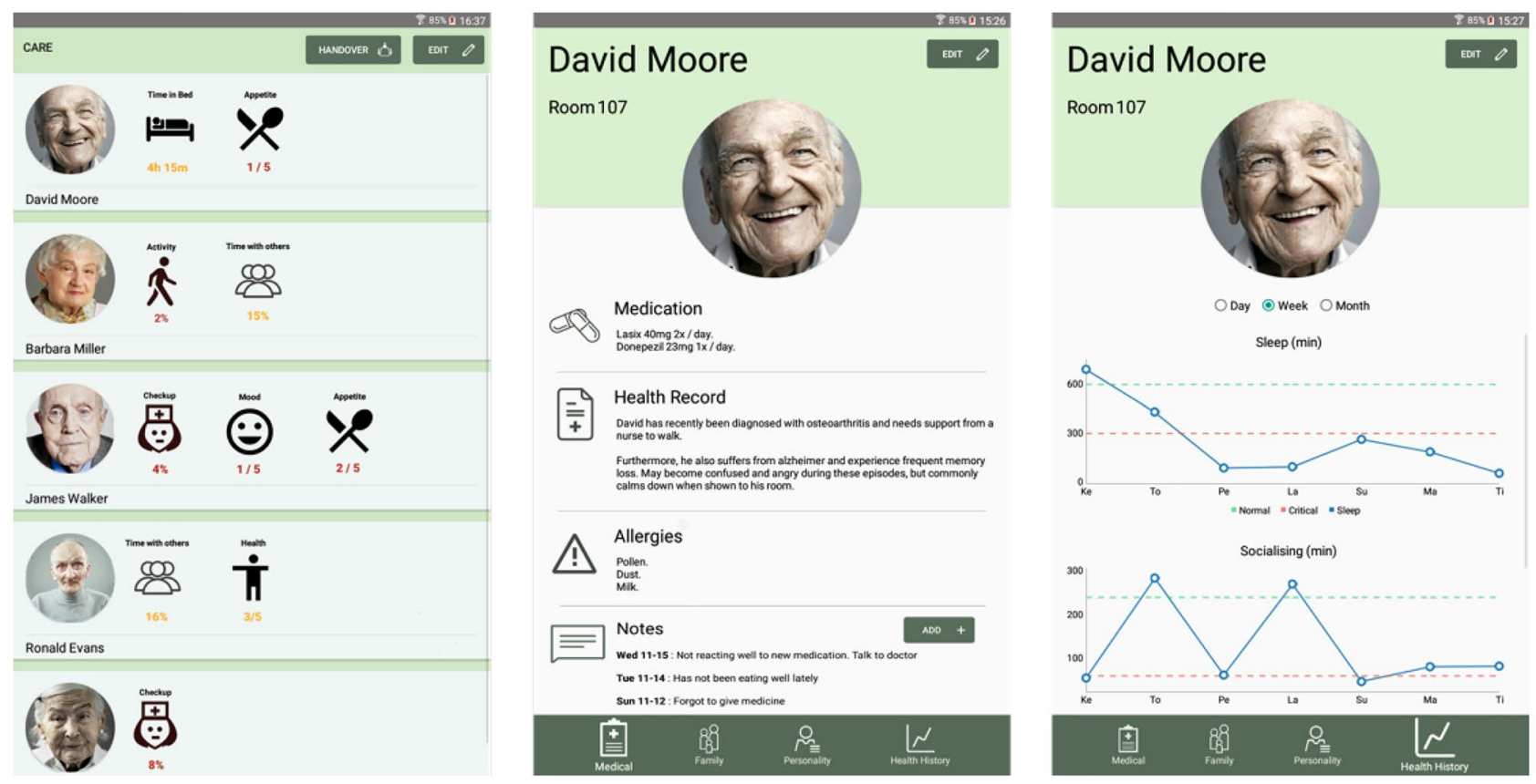

Fig. 1 User Interface of CARE application: Left: Overview of residents' status and care needs. Middle: Information about a resident's medical background. Right: Historical overview of a resident's sensor data 
input an assessment for health, appetite, and mood on a 5-point Likert scale [38].

3 Hardware: The next step was to design the outline of the system that would collect sensor data on the care metrics (e.g., required hardware, interconnections). The nursing home wanted to maintain a home-like setting, so we selected devices which integrated into the environment without being invasive [39].

\section{Integration}

In this section, we start by describing the data management framework and how it interconnects all components of CARE. Following, we expose how the system is integrated into the nursing home. Lastly, we introduce and explain the details regarding the 2-month user study of CARE.

\subsection{Data management framework}

The data management framework was created to facilitate data synchronisation, storage, processing, analysis, visualisation and monitoring. As observed in Fig. 2, the sensors reside at the lowest level. They are responsible for capturing data (e.g., Received Signal Strength Indicator (RSSI), accelerometer) on context changes from nurses, residents, beds, and the general ambience. This data is broadcasted using Bluetooth Low Energy (BLE) to all nearby devices using secure Universally Unique Identifier (UUID) [40]. The Remix Mini (Android PC) devices in the facility are all running a custom Android app, distinct from the tablet app which is used by the nurses (described in section 3). It was created using AWARE, a mobile instrumentation framework for collecting and storing sensor data [41]. The app is a plugin of AWARE, which allows it to run continuously in the background (e.g., it is not killed by operating system for battery saving purposes). The Estimote Software Development Kit (SDK) (authenticated with the Estimote cloud) [42] is integrated with the plugin, enabling it to parse BLE packets from the sensors. The data is first stored locally on the Remix Minis and periodically batch inserted (10K records per batch, at 30-minute intervals) to a MySQL server running on an Amazon Web Service (AWS) server.

The data preprocessing and analytics is done through $\mathrm{R}$ scripts executed on an AWS server. Daily at 10:00, the $R$ scripts calculate summaries (visualised as metrics in the tablet app) using subsections of the data from the previous day previous day and night (24 hours. 06:30 - 06:29). As various infrastructure problems might arise (e.g., WiFi unavailable, syncing crashes) the execution time of the R scripts are set to 3 hours and 30 minutes after the data collection interval for the current day has ended. This allows any Remix Mini which is lagging behind on the sync schedule to recover. It is important that the summaries are calculated using all of the available data to obtain the best possible accuracy. The specific methods utilised to generate the summaries (e.g., algorithms and machine learning approaches) are discussed in the results section, where we take a closer look at the data.

The summaries generated and the data input from nurses utilising the handover functionality (i.e., ranking health, appetite, and mood on a 5-point Likert scale), are stored in the MySQL server on a per resident basis. If the summary or ranking is below a pre-defined threshold, this information is synced to the tablet app and visualised as a care metric. Summaries for sleep, activity, and socialising are synced to the tablet app regardless of their value, as they are shown in the health history graphs. With the number of devices used in our study, we needed an effortless way to ensure that each device was operational (e.g., powered, connected to $\mathrm{Wi}-\mathrm{Fi}$, broadcasting, receiving). Consequently, we designed a Shiny [43] dashboard that provided the researchers with a simple visual overview of the last sync time and transmission details for each unit. Furthermore, we integrated the $\mathrm{R}$ scripts with Slack [44] to monitor the veracity of the daily summaries (e.g., model performance, services running).

\subsection{Setup}

The elderly care facility is roughly 440 square meters and houses 15 residents. An overview of the left housing unit and the common area can be seen in Fig. 3.

As we only had 14 Remix Minis available Fig. 4, we distributed them throughout the building to maximise the sampling coverage. This required some of them to be positioned in resident rooms. Each resident bed was equipped with a Sticker Sensor and most rooms were also equipped with a Beacon Sensor Fig. 4. We had to omit installing hardware in some resident rooms, as certain residents were prone to moving objects or tearing them down. The Wi-Fi network already available in the building was too unreliable, forcing us to install three $4 \mathrm{G}$ routers and four Access Point (AP) extenders to ensure continuous data syncing from the Remix Minis.

Each unit was configured with the appropriate settings (e.g., sampling frequency, broadcasting power, packet configuration). We had conducted several iterations of coding and testing of the software running on the Remix Minis. The virtual IDs and physical labels of the devices were stored in a reference sheet, allowing us to associate incoming data with a specific device. An overview of the bed, resident, and nurse sensors can be seen in Fig. 5, along with examples of how they were positioned on the residents. The sensors were predominately attached to the residents' shoes, but under some circumstances it was affixed to their wheelchair or walking stick if the user regularly relied on these assistive devices. We also provided 


\section{CARE Monitoring}

\section{Cloud Analytics}

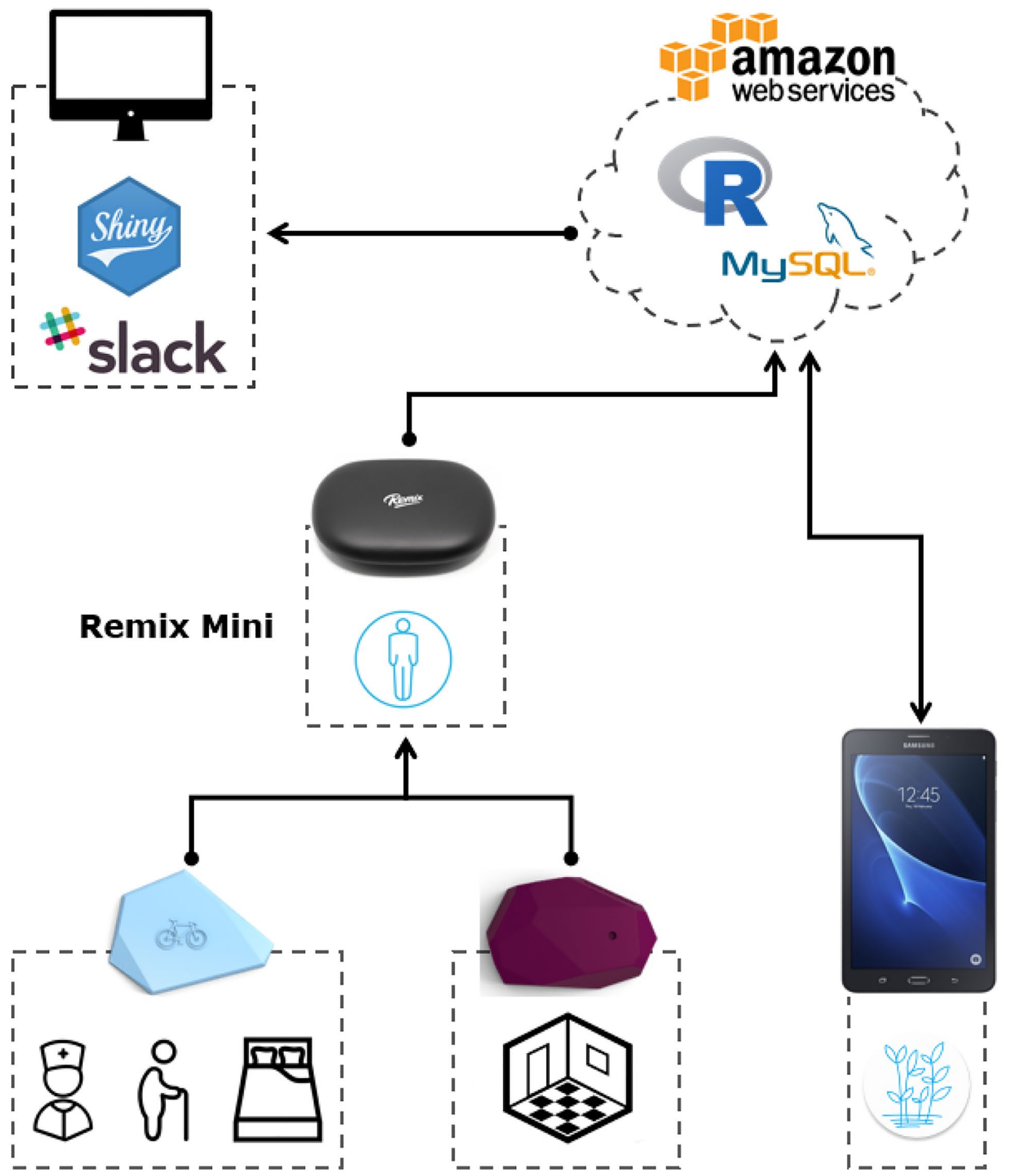

Sticker Sensor

(Accelerometer, RSSI)
Beacon Sensor

(Temperature, ambient light, magnetometer, pressure)

Fig. 2 Overview of the CARE data management framework 
Fig. 3 Overview of one housing unit (left of dashed line), lounge area (top of dashed line) and kitchen (bottom of dashed line). Example hardware positions are marked in red

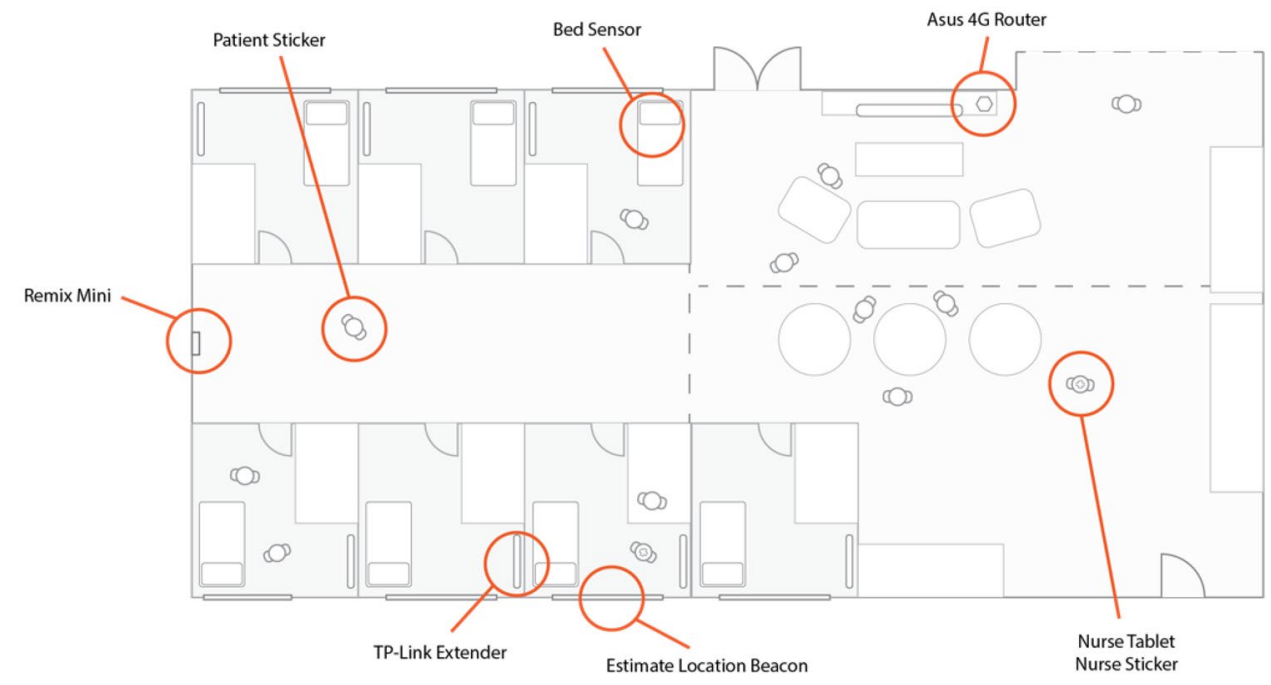

the nurses with a Samsung Galaxy Tab A6 to run the app. The monitoring functionality of CARE was used to detect if a device was erroneous (e.g., disconnected, out of battery, lost), upon which immediate measures were taken to resolve the problem.

\subsection{User study}

Following the successful integration of the sensor infrastructure, we started the 2-month user study with 14 residents and 17 nurses. The management of the nursing home had been involved with the CARE project from the start and was informed of all the details when authorising the user study. An information campaign was conducted to inform all other parties (i.e., nurses, relatives) with regards to the purpose and scope of the project. Participation was optional and residents had to give their consent for wearing the sensor, as it was essential to respect their privacy and rights. The nurses were informed on the necessity of keeping sensor attached to both residents and themselves at all times. In addition, we instructed nurses to pick a sensor from the RF blocking bag upon arrival and return it when leaving the facility. The Radio Frequency (RF) blocking bag ensures that data from unused nurse sensors will not reach a receiver.

We did not track individual nurses (i.e., give a unique sensor to each one) to maintain anonymity and simplify the monitoring of sensors. Given the relatively high number of nurses, it would be challenging to verify if a sensor was dead or the user simply had not been to work lately (i.e., no data activity). During the first month, we only collected data to test models, define thresholds for when care metrics should be shown and ensure that the app was fully integrated with the backend. When the second month commenced, we introduced the app to the nurses and asked them to use it as a tool in their work for the coming month (e.g., check for resident information when necessary, use during handover). The nurses were also responsible for inputting resident-related information to the relevant sections in the application.
Fig. 4 Left: Overview of Remix Minis and Beacon Sensors. Each device is labelled with a unique number depending on its position. Right: Example position of aforementioned devices
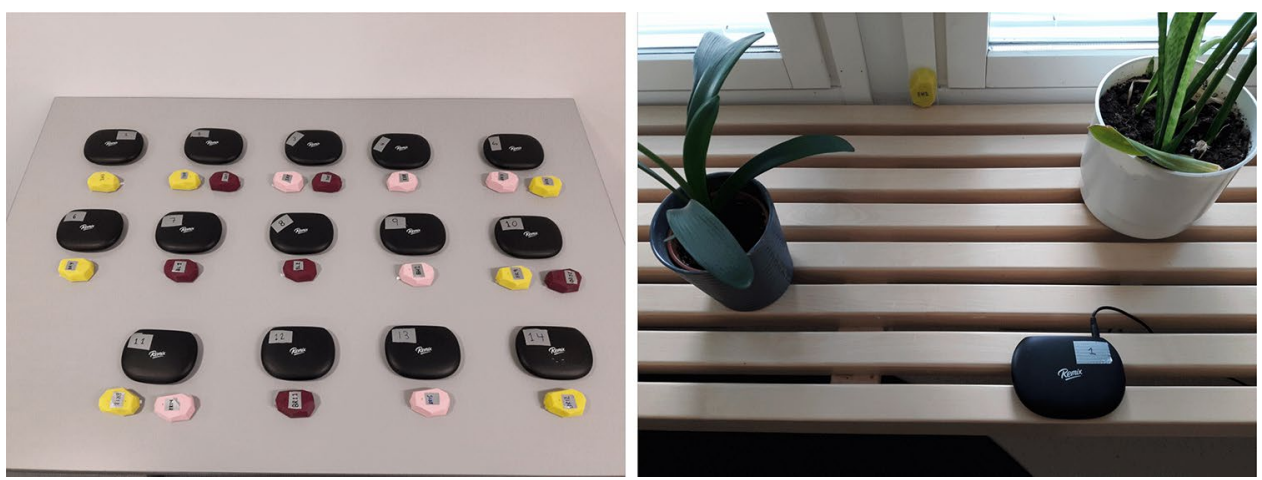

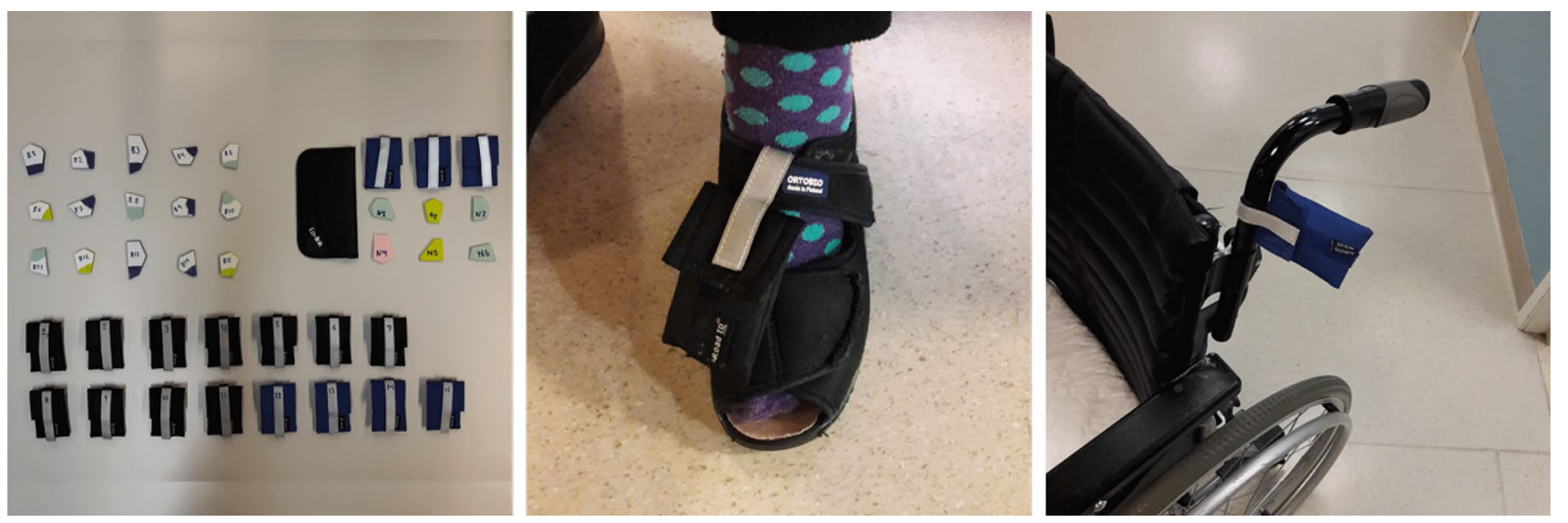

Fig. 5 Left: Overview of bed sensors (top left), resident sensor pouches (bottom), and nurse sensors pouches and RF blocking bag (top right). Middle: Resident wearing sensor on foot. Right: Sensor placement on resident's wheelchair

\section{Results}

In this section, we first present our post-hoc analysis of the data collected from the 2-month user study. We selected a few care metrics which was explored more in detail, to understand the sociocultural context in the care centre. Following, we present the evaluation (i.e., questionnaires and interviews) conducted with the nurses regarding the use of CARE. The findings from the post hoc-analysis are also discussed with the nurses during the interviews.

\subsection{Data analysis}

The final dataset contained a total of 260 million rows from all the sensors (resident, nurse, bed). Graphs presented in this section are summaries of the two-month user study. Depending on the specific analysis, we used a subset of the following features. Timestamp is the time the packet is received by the Remix Mini (identified with Device ID) and Estimote ID is used to determine the unique sensors sending the packet. The acceleration acting on the sensors on each axis (X, Y and $\mathrm{Z}$ ) and the RSSI is also included. As the focus of this paper is on the context of nurses, the elderly and their interactions, we only utilise data from the wearable sensors (i.e., Estimote Stickers) and omit the use of the Estimote Beacons data.

\subsubsection{Proximity}

We used the RSSI values from participants' sensors to determine their position throughout the facility. The nursing home was divided into 19 different geofences and we collected labelled training data consisting of RSSI values from each one. This produced a data frame with 15 features (RSSI-Remix 1, RSSI-Remix2, ... RSSI-Remix14,
Geofence) and 1900 training observations (100 for each geofence) after filtering. This data frame was used to train a Random Forest classifier with 10 -fold cross-validation and the performance is summarised in Table 1 . Proximity is defined as being in the same geofence in our work. The data used in this section is collected from when the residents get up (06:30) until bedtime (20:30).

5.1.1.1 Nurse In Fig. 6. we can observe the proximity to a nurse averaged for all the residents, for a specific time interval. The graph enables an overview of which hours the residents are more exposed to themselves and when the nurses are close by. Between 12:30 and 13:30 the daily handover takes place and this is reflected in the low proximity to a nurse (8\%). In the time intervals $09: 30-11: 30$ and 15:30 - 16:30 the proximity peaks at roughly $22 \%$. This is likely due to meals being served around these times. As a general trend, we notice that nurses are frequently moving around, tending to one resident when many others are left alone.

The average time residents spent in proximity to a nurse did not change significantly between different weekdays. Figure 7 illustrates the differences between residents with regards to their proximity to a nurse. It is evident that there is a considerable difference between residents. While $\mathrm{R} 12$ and $\mathrm{R} 13$ are close to $24 \%, \mathrm{R} 2$ is only in proximity to a nurse for an average of $7.5 \%$ per day. Essentially, this may indicate an imbalance in the amount of care received per resident. This is supported

Table 1 Prediction accuracy of user location (geofence) using Random Forest

\begin{tabular}{lllll}
\hline Algorithm & Accuracy & Precision & Recall & F1 \\
\hline RF & 85.73 & 86.50 & 85.70 & 85.70 \\
\hline
\end{tabular}




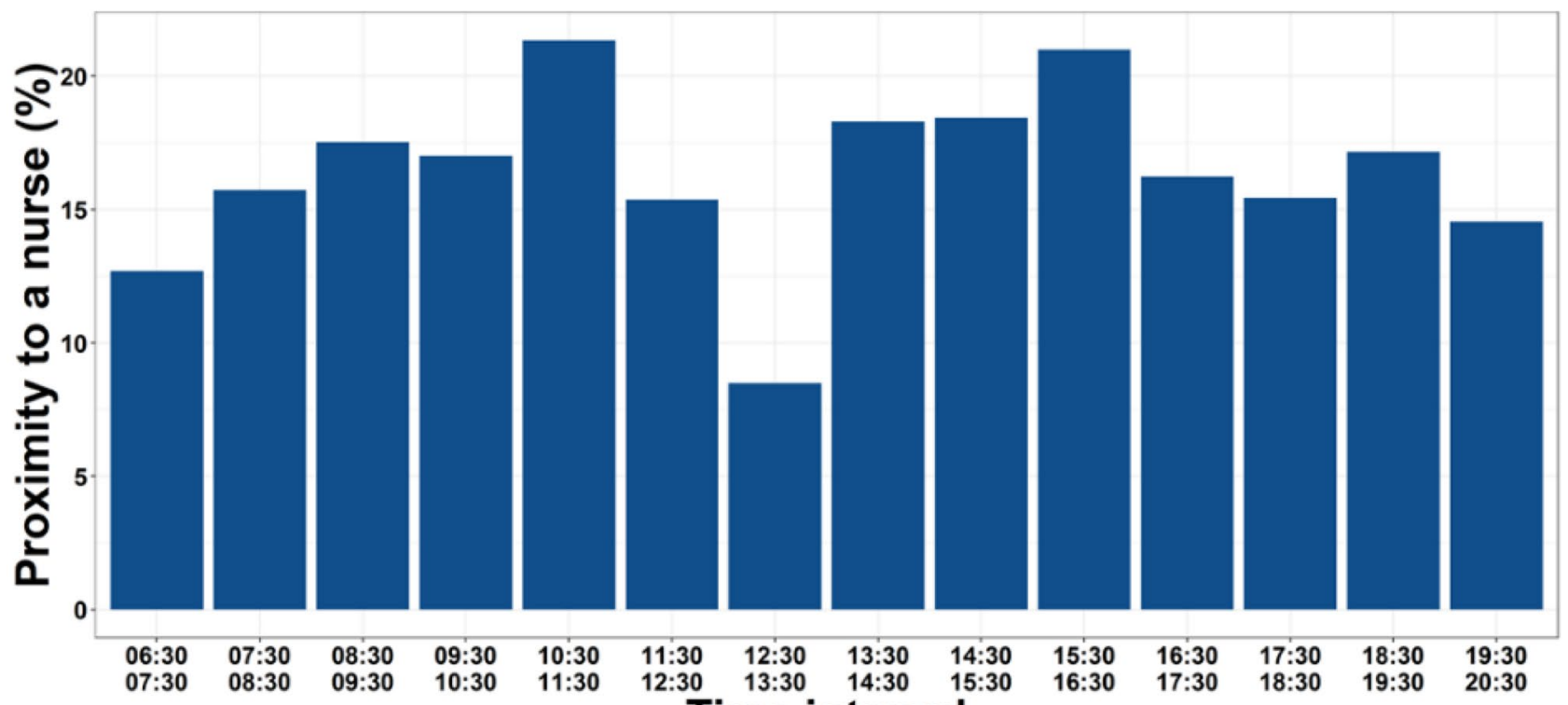

Time interval

Fig. 6 Average \% time in proximity to a nurse per hour

by the statistically significant differences determined by one-way Analysis of Variance (ANOVA) $(\mathrm{F}(14,778)=$ 21.13, $\mathrm{p}<0.001)$.

5.1.1.2 Social The social adjacency matrix Fig. 8 is a visualisation of much time a specific resident spends around other residents. Residents with vertical and hori- zontal rows of dark tiles (e.g., R2, R14, R15) are likely to be isolating themselves in their room. Residents who are spending more times in public areas and around others are annotated with lighter colour ranges (e.g., R7, $\mathrm{R} 8, \mathrm{R} 12$ ). Also, specific pairs of residents (e.g., R1-R8, R7-R8, R14-R15) spend exceedingly more time around each other.

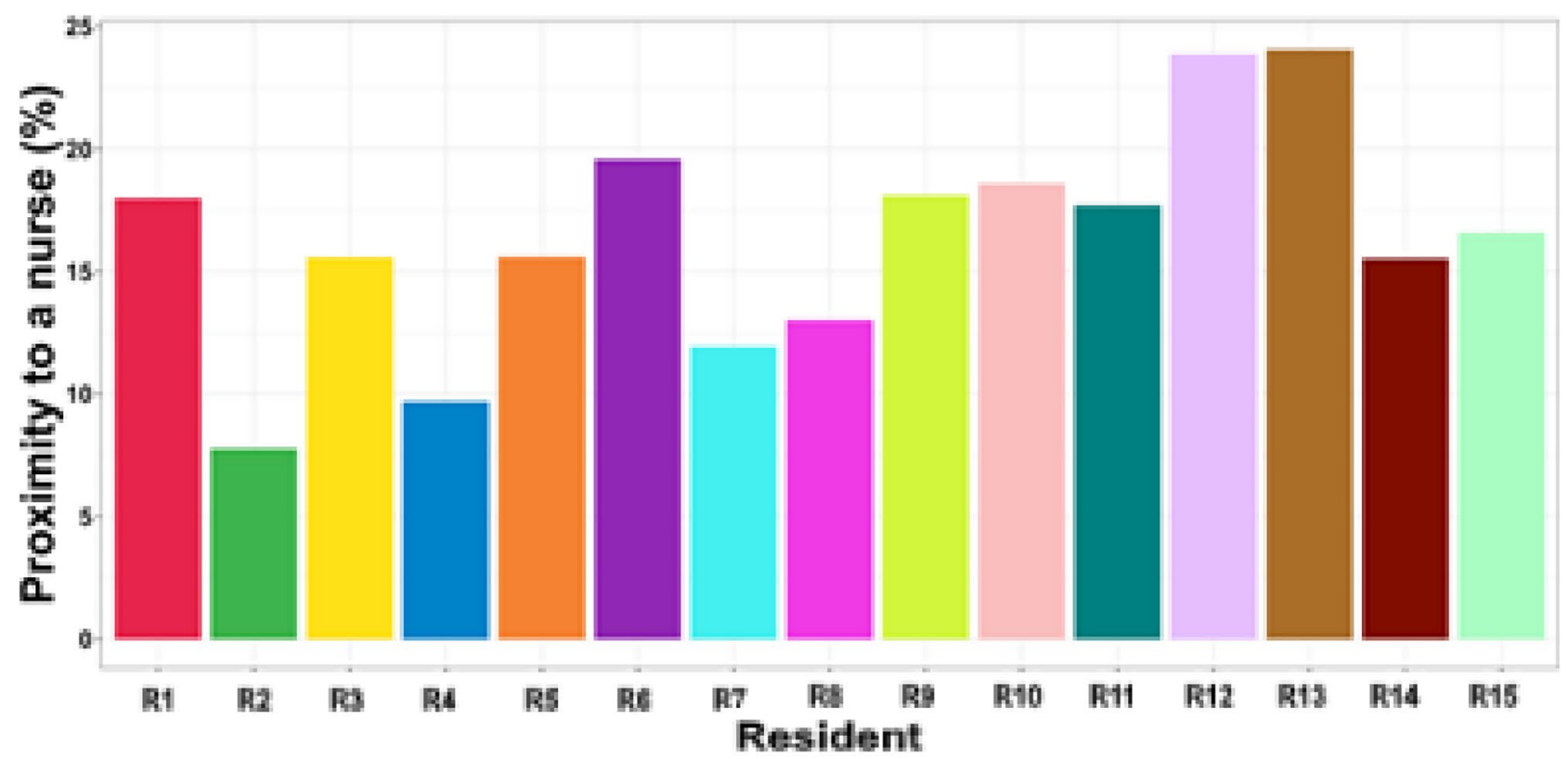

Fig. 7 Average $\%$ time in proximity to a nurse per resident 


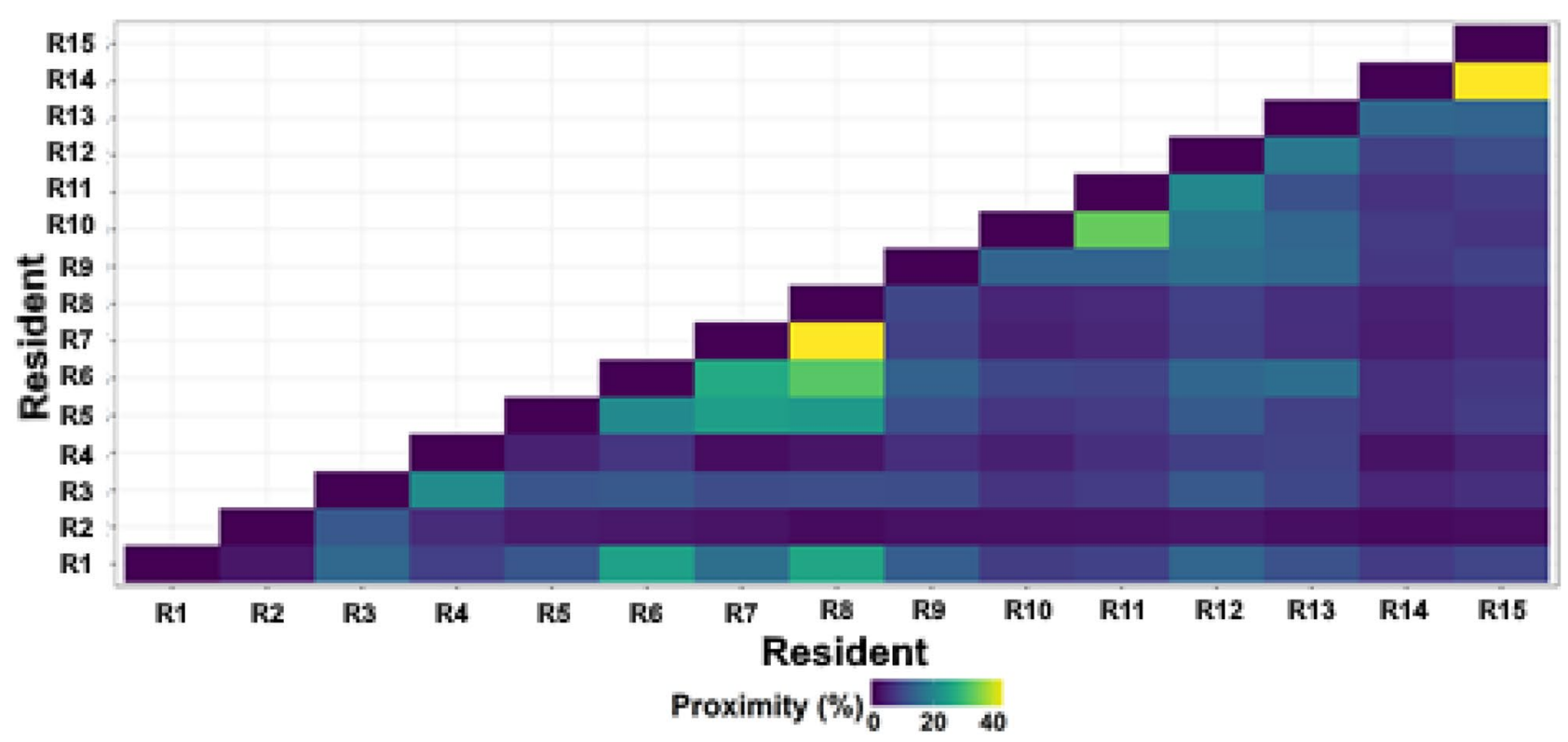

Fig. 8 Social adjacency matrix of residents. The colour scheme represents the level of socialising from dark blue (seldom social proximity) to yellow (frequent social proximity)

\subsubsection{Activity}

To quantify participants amount of daily physical activity, we used the Mean Amplitude Deviation (MAD) metric calculated from raw acceleration data. MAD is efficient for distinguishing light-intensity physical activity from sedentary behaviour $[45,46]$. The metric is device-independent, does not require the data to be filtered in order to correct for gravity and it does not require sensor calibration. We calculated the MAD metric, given in milligravitational $(\mathrm{mg})$ units, on 2-minute epochs of acceleration data. A threshold of $16 \mathrm{mg}$ is used to distinguish between sedentary behaviour and lightintensity physical activity. Similarly, to the previous section, the data interval used is from when the resident wakes up (06:30) until bedtime (20:30).

Figure 9 presents the activity of each resident, while the nurses' values are averaged together. There is considerable diversity in the residents' activity levels and it ranges from very high (R9, R12, R13), high (R3, R6), medium (R10, R11) and low (R14, R15). We can observe that the nurses' average activity is of greater magnitude compared to the residents which indicate substantial physical movement.

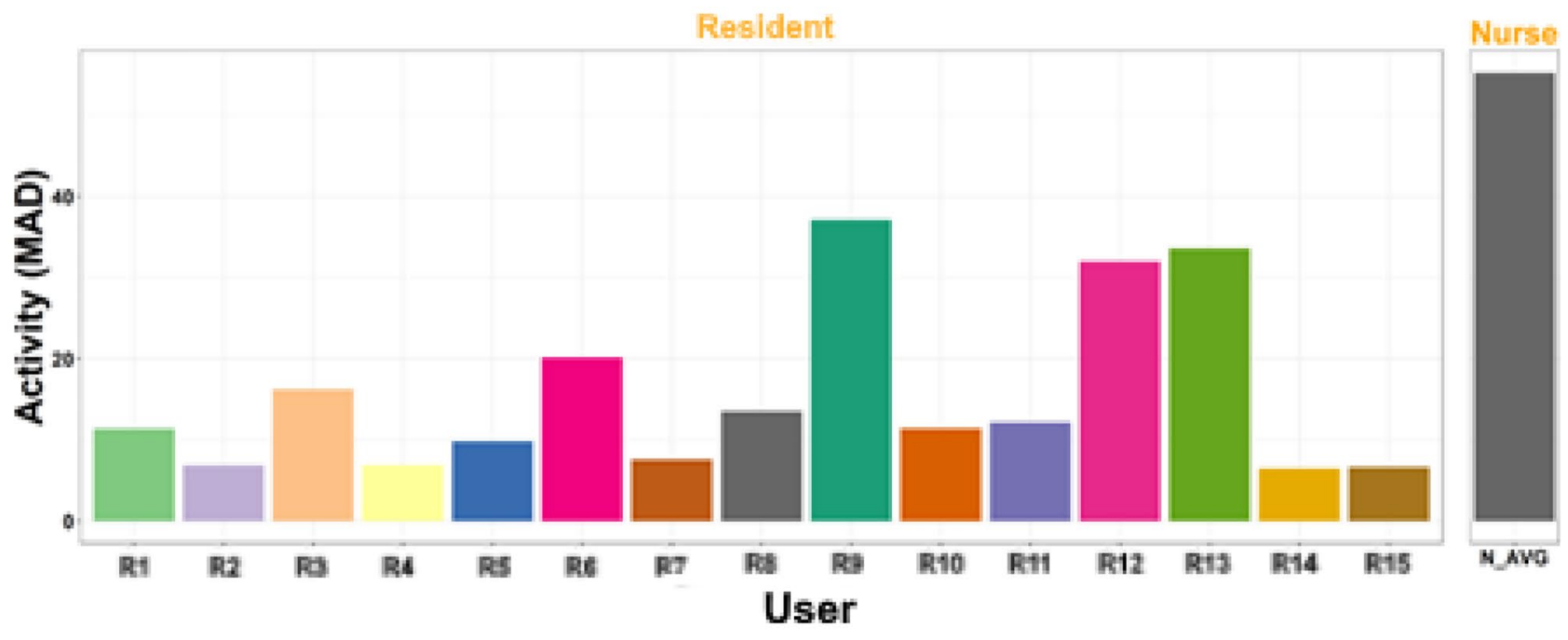

Fig. 9 Activity per user 
The average nurse activity did not change significantly between different weekdays. However, we did see a fluctuating trend from low activity (Wednesday, Saturday) to high (Thursday, Sunday). Nurses spent the most time in proximity to residents during the days with the lowest activity.

\subsubsection{In bed}

The in-bed sensors enable us to capture data on how much time the residents spend in bed. This can be used as a means to recognise underlying health issues in residents, as diseases generally impact sleep. The Sticker Sensor was placed flat under the mattress topper, where it could capture most of the bed orientation through the $\mathrm{x}$ and $\mathrm{y}$ acceleration axis. In contrast to the previous two sections, we rely on data from an entire day (06:30 - 06:29) for inbed analysis. The bed model fitted two k-means clusters [47] to 5-minute epochs of unlabelled accelerometer data. Subsequently, the Ashman's D function from the R package modes [48] was used to check which dimension of the sensor data was more clearly bimodal, corresponding to the two states of the on/off bed detection. The cluster with absolute values closer to 0 corresponded to the empty bed class, and the resident on bed class consisted of the cluster with larger absolute values. This simple device-independent classification algorithm enabled us to automatically train a model for each bed without manually labelled data, despite the differences in the beds, mattresses, sensor locations and orientations, as well as residents' body weights and sleeping habits.
In Fig. 10 we can examine the time in bed for a selection of the residents. R3 has a substantial spread in the upper and lower quartiles, which can indicate some fluctuating sleep behaviour. R4 has the most consistent time measurements with a median close to 10 hours. However, R4 also has a few outliers that might signal an underlying issue these specific days. R7 spent more than 10 hours in bed for $75 \%$ of the days, which is more than the other three participants. In comparison, R11 spent less than 7.5 hours in bed for $50 \%$ of the days and also has the upper quartile with the most variation (from 9 to 16 hours).

\subsubsection{Handover}

The handover data was collected using manual input from nurses during the daily meeting. Residents are assessed on three criteria (appetite, health and mood) using a 5-point Likert scale, as these are hard to measure with the use of sensors. In Fig. 11 we can examine the result for each resident. The count of neutral values (acceptable) is distributed evenly on both sides at $\mathrm{x}=0$. Any negative value (i.e., poor, very poor) will grow the stacked bar chart in the left direction. Likewise, positive values (good, very good) will grow the stacked bar chart in the right direction. We can use this visualisation to identify unhealthy trends in residents' longterm data. For example, R9 is exhibiting signs of illness as the bars are skewed to the left. In contrast, there are residents who seem to do well judging from the right-skewed bars (R5, R7, R12). Some residents have predominantly adequate ratings (R2, R11, R14), but should be monitored to see if their condition worsens over time.

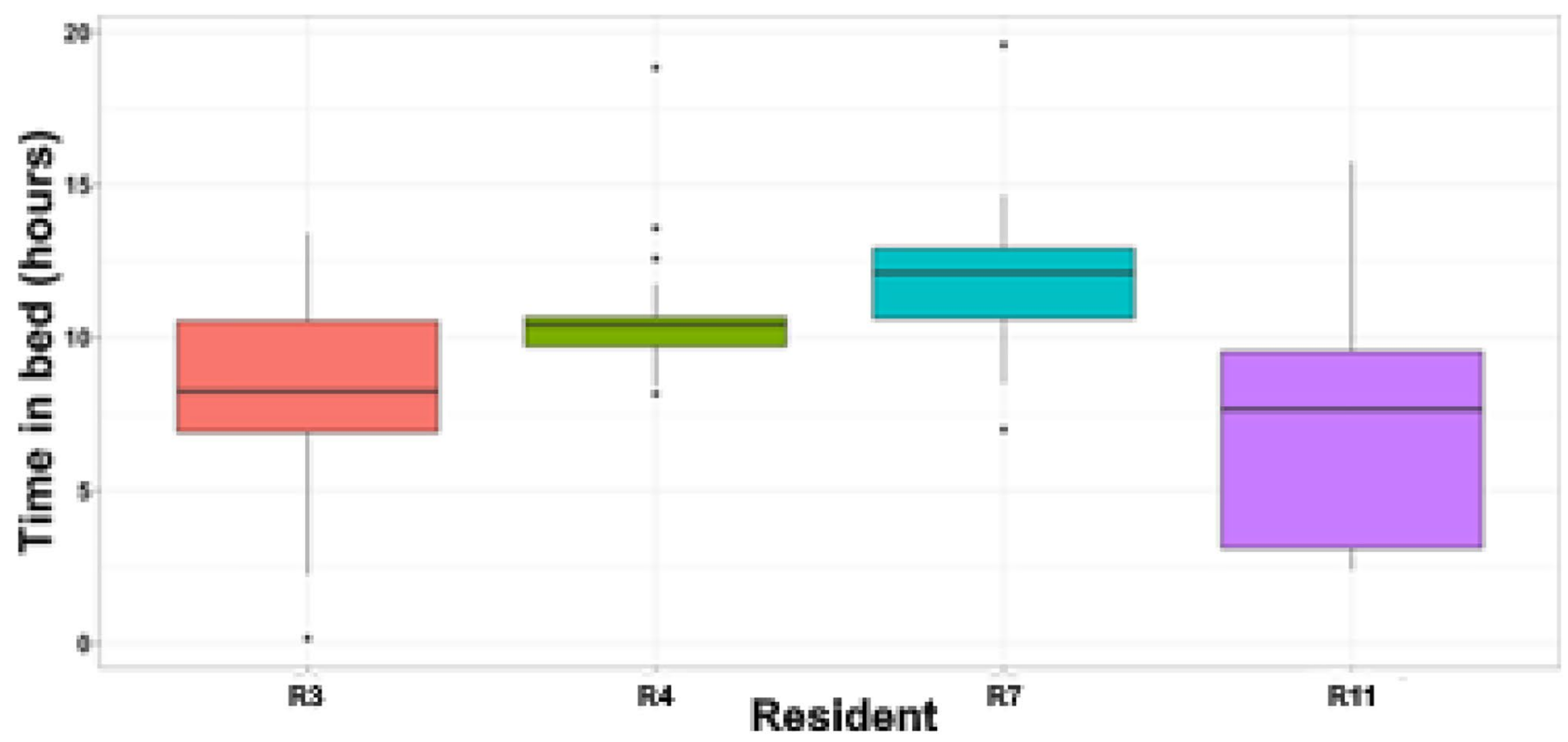

Fig. 10 Boxplot of time spent in bed for four residents 

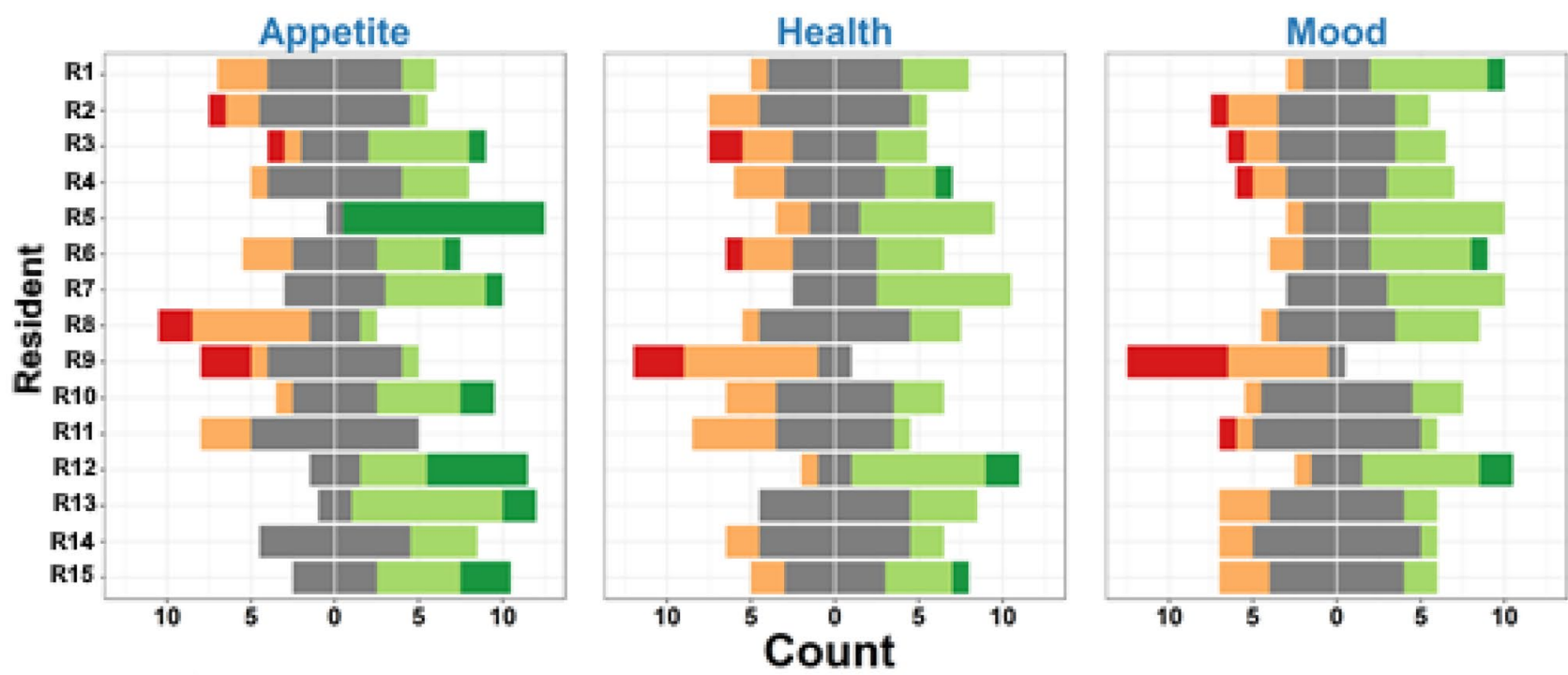

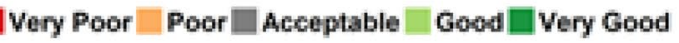

Fig. 11 Visualisation of residents' appetite, health and mood ranked on a 5-point Likert scale

\subsection{Evaluation}

To evaluate CARE, we conducted questionnaires and semistructured interviews with the nurses following the 2-month deployment. This allowed us to understand how the nurses experienced the systems, its benefits and how it integrated with their workflow. A total of eight nurses (sex: 1 male, 7 females; ages: 26 - 56 years old, $\mathrm{M}=33, \mathrm{SD}=10.96$; work experience: 5 - 28 years, $\mathrm{M}=13, \mathrm{SD}=7.31$; roles: 2 nurses, 6 practical nurses) participated in the evaluation.

\subsubsection{Questionnaire}

Elements in the questionnaire were adopted from a few selected theories: Technology trusting disconfirmation [49], Technology satisfaction, Usage continuance intention and Usefulness [50]. These are all theories that have been comprehensively tested in numerous fields and reveal paramount details regarding users' expectations, trust, contentment and willingness for future use of an information system. The complete list of statements and ratings are shown in Table 2. By inspecting the mean score, we see that all conditions are rated above the median (4), except condition 5 . While nurses did not think the app increase their performance (e.g., complete tasks faster), the ratings for condition 3,6, and 8 indicate that they found value in using the tool. Furthermore, the app received positive ratings for its ability to identify long-term trends using the health history tab (condition 7). Overall, the nurses were satisfied with their use of CARE (condition 2) and displayed a strong willingness to continue using the system in the future (condition 4). This indicates that the introduction of

Table 2 Questionnaire results from the CARE evaluation

\begin{tabular}{|c|c|c|c|c|}
\hline \# & Statement & Scale & Mean & SD \\
\hline 1 & I would rate my level of experience with smartphone applications as & $\begin{array}{l}\text { (1) No experience - } \\
\text { (7) Extensive experience }\end{array}$ & 5.87 & 0.83 \\
\hline 2 & I am ___ with my use of CARE & $\begin{array}{l}\text { (1) Extremely Dissatisfied - } \\
\text { (7) Extremely Satisfied }\end{array}$ & 4.87 & 0.99 \\
\hline 3 & The app has the functionality and features required for improving patient care & $\begin{array}{l}\text { (1) Strongly Disagree - } \\
\text { (7) Strongly Agree }\end{array}$ & 4.37 & 1.30 \\
\hline 4 & I intend to continue using CARE to access patient-related information after this study & " & 4.75 & 1.16 \\
\hline 5 & Based on your experience with CARE: it improved my performance & “" & 3.75 & 0.89 \\
\hline 6 & Based on your experience with CARE: it was useful & “' & 4.5 & 0.75 \\
\hline 7 & I can identify long-term behavioural and health changes in patient & ', & 5.5 & 1.07 \\
\hline 8 & I am satisfied with the amount of available information describing patients' context & “' & 5 & 1.07 \\
\hline
\end{tabular}


the technology in the care facility succeeded and that user acceptance was achieved among the users.

\subsubsection{Interviews}

We conducted semi-structured interviews with the nurses to complement the quantitative data and present a more comprehensive evaluation. Groups of 2-3 nurses were interviewed at a time to foster discussion amongst participants. The questions were open-ended and probed the nurses on the usage of CARE (i.e., usability, useful aspects, workflow integration). We compressed the resulting set of data to components that related to the project scope. Then emerging themes were established from this subset of qualitative information, which again were grouped by the most significant common themes.

5.2.2.1 System usability Most aspects regarding the usability of the app had been addressed in the preliminary work. We were still interested in how the nurses experienced it throughout the study. The result was primarily in line with previous findings.

"The app is easy to use, clear and simple. It was quick to fill in the information and did not take unnecessary time."

As the system we developed proposes a completely new way for the nurses to work, they expressed that it took some time to consolidate a usage pattern.

"It was a bit difficult to always remember to have the application (tablet) with us during the meeting and we occasionally forgot it. We usually filled in the information later if this happened."

The nurses stated that use of the system got better through practice and gradually it became a greater part of their routine.

"We did not find the experiment to be cumbersome, as we were just required to remember to keep the sensors with us. We did forget a few times in the beginning, but after that it became almost automatic."

We received positive feedback on the usage. As the study progressed, nurses got a better understanding of CARE.

"I was really sceptical of the system, the sensors and the information the application could provide us at first. But this experiment and its results have been a very positive surprise for me."

5.2.2.2 Technology adoption An important aspect related to introducing new technology in a healthcare environment is how the users incorporate it into their daily work. Namely, we were interested in how nurses would adopt the use of the app. Nurses confirmed that the handover functionality was utilised on a frequent basis as intended.

"Logging the information during the handover, as a group, facilitated a detailed assessment."

We were also interested in other use cases in which the app could provide value. Through discussions with the participants, we discovered that they appreciated how the app acted as a hub of information.

"It is really useful to share this information if someone has been sick or can not make it to the meeting. Also, if we were unable to distribute resident-related information with other nurses, they can find it in the app."

We noted one specific use case for the longitudinal data described by a nurse.

"I reviewed the patient health history data when returning from a few days break. This allowed me to detect potential issues that I could ask colleagues to clarify."

A nurse also mentioned how CARE might introduce new ways of working in a preventive and data-driven manner.

"We could examine plots to detect changes or consult them when some new behaviour has been detected in the patient."

5.2.2.3 Patient awareness A fundamental part of CARE is increasing nurses' awareness to residents' needs. For example, by exposing an uneven level of care received by residents. The nurses were not aware that such an issue was present in the care facility.

"We do not quantify how much time we spend with the residents and it is difficult to assess without the use of any tool. Some of the aspects related to time spent with each resident were quite surprising, especially how little time we ended up spending with the residents who mostly stay in their rooms."

The nurses reflected on these results, and also debated whether they should change their workflow to increase the time they spend around more passive residents.

"We do spend very little time with some of the residents, which was surprising. We probably should do more than just the bare essentials with some of them."

The data gathered from the handover sessions also provided insights into how the residents were doing on a dayto-day basis.

"I found it very interesting to see how appetite, health and mood fluctuated at different days." 
We also received feedback on how gradual and long-term changes in resident behaviour would be easier to notice with CARE's health history data.

"If a patient's mood starts to change slowly and gradually over a long period, it could be hard or impossible to register without this type of tool."

\section{Discussion}

\subsection{Designing CARE}

Previous systems which target independent living have functionality that can be superfluous (e.g., telehealth, assisted living) in a nursing home [34]. Also, the limited body of work which exists for assistive sensor systems in nursing homes indicate a need for a tool that has a more extensive range of utility [8, 9, 30, 35-37]. When developing CARE, we specifically focused on addressing these concerns and integrate a multitude of services that can support caregiving. Hence, it was essential to sense the context of both nurses and residents to understand their interactions, instead of selecting one [29, 30].

CARE was designed to be non-obtrusive and follow the vision of creating a familiar and home-like living space for the residents, in contrast to a hospital setting. This involves not placing invasive technology (e.g., large and obtrusive sensors) on either the resident or nursing staff. We decided against using a hand-worn sensor to measure biosignals (e.g., heart rate, galvanic skin response, body temperature), as it would enforce additional tasks upon the staff. The device would have to be recharged every two-days and it might upset the residents to have it placed on their wrist. The smartphone is also a popular and powerful tool to monitor human activity [31]. Personal healthcare applications running on smartphones can track important and useful data for health diagnostics [32]. We explored this option, but the form factor of smart devices is not suitable for our utilisation (e.g., large, fragile, costly). CARE mainly consists of units with low cost, minimal maintenance requirements (durable sensors, long battery lifetime) and small form factor. This is to avoid the infrastructure from interfering with the nurses' workflow.

The mobile CARE app acts as an interface between the sensor data collected and the nurses. It can be accessed insitu when required, in comparison to the existing PC-based information system which was described as inconvenient and uninformative. It is intended to be easy to use and the goal is to enable a better care service by raising awareness to the daily and long-term needs of the elderly. As we occasionally experienced some issues with the hardware infrastructure (e.g., data not syncing, Wi-Fi down), the monitoring dashboard was useful to detect these events and allowed us to promptly solve the issue.

\subsection{Quantified care}

Big data analytics is a valuable resource for nurses, as it empowers them with vital patient insights from vast data sources [51]. Similarly, CARE utilises a plethora of wellbeing metrics (e.g., activity, time in bed, social and nurse proximity) to inform the nurses on the status of patients. Both daily and long-term summaries are visualised, so dangerous trends can be identified and prevented. The proximity data was used to estimate the level of personal care and attention a patient is receiving. While a nurse is not always actively caring for a patient located in the same geofence (e.g., tending to other patients), they can quickly intervene when an event occurs (e.g., patient falls) and stay more aware of their needs. The activity measurements can be used to determine if patients get enough physical exercise. The data can also reveal the healthier and independent individuals. Analysing nurses' activity can indicate the workload they experience, as their tasks require a high level of mobility and are physically demanding. Also, the in-bed and handover assessments can expose underlying health issues experienced by patients.

The data analysis uncovered interesting trends in the sociocultural context, such as an uneven distribution of care. CARE received positive feedback for its ability to enable evidence-based care. The nurses expressed that some aspects of the care context (e.g., patient not socialising, health declining) were almost impossible notice on a day-to-day basis without the health history graphs. The nurses also wanted to improve their workflow based on the new information they were empowered with. In future nursing homes, we could standardise thresholds for the various care metrics, to ensure a high level of care across different care institutions.

\subsection{Assisting nursing workflow}

The increasing demand for elderly care services causes an exhausting work environment and high turnover rates [1]. During the time we spent at the nursing home, we witnessed an influx of new personnel who are not familiar with the residents or their needs. Nurses are therefore required to spend a considerable amount of time instructing and informing newcomers, resulting in less time for caregiving. This need is not exclusive to newcomers; regular staff also needs quick and effortless day-to-day resident information. Checking an (inconveniently placed) computer for medication and medical notes is a breakdown (i.e., interruption) to their workflow. As CARE automatically senses residents' contextual data over extended periods, nurses are provided with a historical overview 
of residents' status. CARE also include qualitative data (e.g., resident background, likes, dislikes) and can be an invaluable tool to speedily consult information for learning, planning and decision making. We observed a need for an accurate in-situ method for medication identification, but we did not have time to implement this functionality. While ICT have several benefits in a medical context, many implementations fail [21]. A common reason for low adoption rates is nurses' critique against changes in established workflow (e.g., cumbersome use, additional labour). We utilise a user-driven methodology in the design of CARE, to complement the current workflow and not replace it. Using the app is optional and a majority of the available information is automatically collected.

Nurses' assignments are often brief, required substantial mobility and the order change unexpectedly due to evolving temporal and spatial context [23]. Consequently, analytical thinking, decision making and sharing of information can be impaired in such a hectic environment, leading to a decline in the care quality. We designed CARE to empower nurses with the data that is otherwise lost, to help alleviate these issues and maintain an overview of residents' needs. For instance, the handover mode enables a structured agenda and in-depth resident data for this critical daily event. Trends over time can be visually inspected and discussed amongst the meeting members, to predict future illness and to reflect on previous situations. The nurses seemed to implement the app as a part of their workflow and the positive ratings from the questionnaire indicated a willingness for future use of CARE.

\section{Limitations}

We based our system requirements on one nursing home. While this nursing home is a part of a larger care foundation which shares care practices, it would be interesting to investigate the work environment in multiple facilities. We must acknowledge that our data collection is not perfect. External factors such as interference, absorption, and diffraction impact radio signals, resulting in varying RSSI. This is reflected in the accuracy of geofence prediction with RF. This could be improved by increasing the transmitting power of the sensors (also causing a higher battery drain) and install more Remix Minis (receivers). We did not pursue any of the two alternatives, as acquiring more hardware would lead to increased project costs. Also, the resident had different types of beds (e.g., motorised, air loss mattress) which at times led to noisy data. We did not have complete datasets for each user during a day (i.e., gaps exist in data collection lasting from seconds to minutes). This is caused by different factors. For instance, BLE undirected advertising does not implement retransmission, and packets containing errors are dropped [52]. Additionally, certain areas of the facility had worse coverage depending on how close the nearest Remix Mini was. Therefore, we choose to report the percentage of total time available in some of the data analysis graphs rather than an exact 24-hour scale.

\section{Conclusion}

In this work we have presented CARE, a context-aware tool for nurses in nursing homes. The system incorporates a sensor infrastructure which measures residents' and nurses' behaviour and a data management platform which generates analytical insights. Nurses can access this data, other qualitative resident information and functions that support their work through an Android app. The primary objective of CARE is to increase awareness to residents' needs, enabling informed decision making and an enhanced care service. We evaluated our system in a 2-month long user study at a local nursing home. The result indicates that CARE can improve the care service and reveal important insights on the health and care received by the elderly residents. The system also received positive feedback from questionnaires and interviews conducted with nurses, which implies that the nurses would like to continue using the system.

Funding Open access funding provided by University of Oulu including Oulu University Hospital. This work is partially funded by the Academy of Finland (Grants 286386-CPDSS, 285459-iSCIENCE, 304925-CARE, 313224-STOP, 316253-SENSATE), and Marie Skłodowska-Curie Actions (645706-GRAGE).

Code availability Available at https://github.com/sklakegg/EstimoteAnalysis-R.

\section{Compliance with ethical standards}

Conflicts of interest On behalf of all authors, the corresponding author states that there is no conflict of interest.

Open Access This article is licensed under a Creative Commons Attribution 4.0 International License, which permits use, sharing, adaptation, distribution and reproduction in any medium or format, as long as you give appropriate credit to the original author(s) and the source, provide a link to the Creative Commons licence, and indicate if changes were made. The images or other third party material in this article are included in the article's Creative Commons licence, unless indicated otherwise in a credit line to the material. If material is not included in the article's Creative Commons licence and your intended use is not permitted by statutory regulation or exceeds the permitted use, you will need to obtain permission directly from the copyright holder. To view a copy of this licence, visit http://creativecommons.org/licenses/by/4.0/. 


\section{Reference}

1. Hasson H, Arnetz J E. Nursing staff competence, work strain, stress and satisfaction in elderly care: a comparison of home-based care and nursing homes. J Clin Nurs. 2008;17(4):468-81.

2. Marie R Squillace, Anita Bercovitz, Emily Rosenoff and Robin Remsburg. An exploratory study of certified nursing assistants' intent to leave. US Department of Health and Human Services, Assistant Secretary for Planning and Evaluation, Office of Disability, Aging and Long-Term Care Policy; 2008.

3. Moran E B, Tentori M, Gonzalez V M, Favela J, MartinezGarcia A I. Mobility in hospital work: towards a pervasive computing hospital environment. Int $\mathrm{J}$ Electron Healthc. 2006;3(1):72-89.

4. Klakegg S, van Berkel N, Visuri A, Luo C, Goncalves J, Hosio S, Huttunen H, Ferreira D. Informing Caregivers Through an Assistive Tool: An Investigation of Elderly Care Metrics. Sunderland, UK: In British HCI; 2017.

5. World Health Organization. Action on patient safety: high $5 \mathrm{~s}$. Geneva, Switzerland: Author. Retrieved October, 22. 2011; 2006

6. Manser T, Foster S. Effective handover communication: an overview of research and improvement efforts. Best Pract Res Clin Anaesthesiol. 2011;25(2):181-91.

7. Aloulou H, Mokhtari M, Tiberghien T, Biswas J, Phua C, Lin Jin Hong Kenneth, Yap Philip. Deployment of assistive living technology in a nursing home environment: methods and lessons learned. BMC Med Inform Decis Mak. 2013;13(1):42.

8. Chang YJ, Chen $\mathrm{CH}$, Lin LF, Han RP, Huang WT, Lee GC. Wireless sensor networks for vital signs monitoring: Application in a nursing home. Int J Distrib Sens N. 2012;8(11):685107.

9. Huang C, Chiang C, Guan-Chun Chen S, Hsu W, Chan C. Fall detection system for healthcare quality improvement in residential care facilities. J Med Biol Eng. 2010;30(4):247-52.

10. Memon M, Wagner SR, Pedersen CF, Beevi FHA, Hansen FO. Ambient assisted living healthcare frameworks, platforms, standards, and quality attributes. J Sens. 2014;14(3):4312-41.

11. Hall A, Wilson CB, Stanmore E, Todd C. Implementing monitoring technologies in care homes for people with dementia: A qualitative exploration using Normalization Process Theory. Int J Nurs Stud. 2017;72:60-70.

12. Webster G, Hanson V L. Technology for Supporting Care Staff in Residential Homes. ACM Trans Access Comput. 2014;5(3):8.

13. Bjorn Lindgren. The Rise in Life Expectancy, Health Trends among the Elderly, and the Demand for Care-A Selected Literature Review, National Bureau of Economic Research; 2016.

14. Colby SL, Ortman JM. Projections of the size and composition of the US population: 2014 to 2060: Population estimates and projections. 2017

15. OECD Indicators. Health at a Glance 2011. OECD Indicators, OECD Publishing, Paris. https://doi.org/10.1787/health_ glance-2015-en. Accessed February, 15. 2016. 2015

16. A Universal Truth. No Health without a workforce. World Health Organisation (WHO) Report. 2013

17. Engström M, Ljunggren B, Lindqvist R, Carlsson M. Staff satisfaction with work, perceived quality of care and stress in elderly care: psychometric assessments and associations. Journal of Nursing Management. 2006;14(4):318-28.

18. Tzeng H. The influence of nurses' working motivation and job satisfaction on intention to quit: an empirical investigation in Taiwan. J Nurs Stud. 2002;39(8):867-78.

19. European Central Bank. Demographic change in the euro area: projections and consequences European Central Bank Monthly Bulletin. 2006;49-64
20. K Giannakouris. Eurostat: Ageing Characterises the Demographic Perspectives of the European Societies. 2008. Page: http:// ec.europa.eu/eurostat

21. Marcia M Ward, Smruti Vartak, Tammy Schwichtenberg and Douglas S Wakefield. Nurses' perceptions of how clinical information system implementation affects workflow and patient care. CIN: Computers, Informatics, Nursing. 2011;29(9):502-511.

22. Cornell Paul, Herrin-Griffith Donna, Keim Courtney, Petschonek S, Sanders A M, D'mello S, Golden T W, Shepherd G. Transforming nursing workflow, part 1: the chaotic nature of nurse activities. J Nurs Adm. 2010;40(9):366-73.

23. Vardaman J M, Cornell P T, Clancy T R. Complexity and change in nurse workflows. J Nurs Adm. 2012;42(2):78-82.

24. Marina Kobayashi, Susan R Fussell, Yan Xiao and F Jacob Seagull. Year. Work coordination, workflow, and workarounds in a medical context. In CHI'05 extended abstracts on Human factors in computing systems, ACM, 1561-1564.

25. British Medical Association. Safe handover: safe patients. Guidance on clinical handover for clinicians and managers. London: BMA, 2004;7:141

26. Payne S, Hardey M, Coleman P. Interactions between nurses during handovers in elderly care. J Adv Nurs. 2000;32(2):277-85.

27. Leotsakos A, Zheng H, Croteau R, Loeb J M, Sherman Heather, Hoffman Carolyn, Morganstein Louise, O'leary Dennis, Bruneau Charles, Lee Peter. Standardization in patient safety: the WHO High 5 s project. International journal for quality in health care. 2014;26(2):109-16.

28. Abbate S, Avvenuti M, Corsini P, Light J, Vecchio A. Monitoring of human movements for fall detection and activities recognition in elderly care using wireless sensor network: a survey. In Wireless Sensor Networks: Application-Centric Design, InTech; 2010.

29. Hsu H, Chen C. RFID-based human behavior modeling and anomaly detection for elderly care. Mobile Information Systems. 2010;6(4):341-54.

30. Inoue S, Ueda N, Nohara Y, Nakashima N. Recognizing and Understanding Nursing Activities for a whole day with a big dataset. Journal of Information Processing. 2016;24(6):853-66.

31. Lee Y, Jeong WS, Yoon Gilwon. Smartphone-based mobile health monitoring. Telemedicine and e-Health. 2012;18(8):585-90.

32. Pang $\mathrm{Wu}$, Huan-Kai Peng, Jiang Zhu and Ying Zhang. Year. Senscare: Semi-automatic activity summarization system for elderly care. In International Conference on Mobile Computing, Applications, and Services, Springer, 1-19.

33. Aanesen M, Lotherington AT, Olsen F. Smarter elder care? A cost-effectiveness analysis of implementing technology in elder care. J Health Inform. 2011;17(3):161-72.

34. Kvedar J, Coye MJ, Everett W. Connected health: a review of technologies and strategies to improve patient care with telemedicine and telehealth. Health Affairs. 2014;33(2):194-9.

35. Nava-Muñoz S, Morán A L. CANoE: a context-aware notification model to support the care of older adults in a nursing home. J Sens. 2012;12(9):11477-504.

36. Merilahti J, Viramo P, Korhonen I. Wearable monitoring of physical functioning and disability changes, circadian rhythms and sleep patterns in nursing home residents. IEEE J Biomed Health. 2016;20(3):856-64.

37. Cahill J, McLoughlin S, Wetherall S. The Design of New Technology Supporting Wellbeing, Independence and Social Participation, for Older Adults Domiciled in Residential Homes and/or Assisted Living Communities. Technologies. 2018;6(1):18 
38. Simon Klakegg, Niels van Berkel, Aku Visuri, Chu Luo, Jorge Goncalves, Simo Hosio, Hanna-Leena Huttunen and Denzil Ferreira. Year. Informing Caregivers Through an Assistive Tool: An Investigation of Elderly Care Metrics. In Proceedings of the 31st British Human Computer Interaction Conference, BCS Learning and Development Ltd.

39. Simon Klakegg, Niels van Berkel, Aku Visuri, Hanna-Leena Huttunen, Simo Hosio, Chu Luo, Jorge Goncalves and Denzil Ferreira. Designing a context-aware assistive infrastructure for elderly care. In Proceedings of the 2017 ACM International Joint Conference on Pervasive and Ubiquitous Computing and Proceedings of the 2017 ACM International Symposium on Wearable Computers, Maui, Hawaii, ACM, 2017:563-568. https://doi. org/10.1145/3123024.3124403

40. Jess Anderson. Are Estimote beacons secure? How does Secure UUID work?, Estimote. 2017.

41. Ferreira D, Kostakos V, Dey AK. AWARE: Mobile Context Instrumentation Framework. Frontiers in ICT. 2015;2(6):1-9. https:// doi.org/10.3389/fict.2015.00006.

42. Estimote, Intro to Estimote APIs. Accessed from https://developer. estimote.com/

43. W Chang, J Cheng, JJ Allaire, Y Xie and J McPherson. shiny: Web application framework for R [Computer software]. 2017. http:// CRAN.R-project.org/package=shiny $($ R package version1.0.0)

44. slackr, Package 'slackr'. Accessed from https://cran.r-project.org/ web/packages/slackr/slackr.pdf

45. Bakrania K, Yates T, Rowlands A V, Esliger D W, Bunnewell S, Sanders J, Davies M, Khunti K, Edwardson C L. Intensity thresholds on raw acceleration data: euclidean norm minus one (ENMO) and mean amplitude deviation (MAD) approaches. PloS one. 2016;11(10):e0164045.
46. Vähä-Ypyä H, Vasankari T, Husu P, Suni J, Sievänen H. A universal, accurate intensity-based classification of different physical activities using raw data of accelerometer. Clin Physiol Funct Imaging. 2015;35(1):64-70.

47. Hartigan JA, Wong MA. Algorithm AS 136: A k-means clustering algorithm. Journal of the Royal Statistical Society. Series $C$ (Applied Statistics). 1979;28(1):100-108

48. Deevi S. Find the Modes and Assess the Modality of Complex and Mixture Distributions. CRAN: Especially with Big Datasets; 2016.

49. Harrison McKnight D, Choudhury V, Kacmar C. Developing and validating trust measures for e-commerce: An integrative typology. Inf Syst Res. 2002;13(3):334-59.

50. Bhattacherjee A, Premkumar G. Understanding changes in belief and attitude toward information technology usage: A theoretical model and longitudinal test. MIS quarterly. 2004;229-254

51. Murdoch T B, Detsky A S. The inevitable application of big data to health care. Jama. 2013;309(13):1351-2.

52. Evgeny Tsimbalo, Xenofon Fafoutis, Evangelos Mellios, Mo Haghighi, Bo Tan, Geoffrey Hilton, Robert Piechocki and Ian Craddock. Year. Mitigating packet loss in connectionless Bluetooth Low Energy. In Internet of Things (WF-IoT), IEEE 2nd World Forum on, IEEE, 2015;291-296. 